European

Thyroid Journal
Eur Thyroid J 2019;8:59-63

DOI: $10.1159 / 000496924$
Received: September 10, 2018

Accepted after revision: January 14, 2019

Published online: March 12, 2019

\title{
Insulin Sensitivity and Beta-Cell Function in Graves' Disease and Their Changes with the Carbimazole-Induced Euthyroid State
}

\author{
Nandhini Lakshmana Perumal Jayakumar Selvi Kalyani Sridharan \\ Jayaprakash Sahoo Sadishkumar Kamalanathan \\ Department of Endocrinology and Metabolism, Jawaharlal Institute of Postgraduate Medical Education and \\ Research (JIPMER), Puducherry, India
}

\section{Keywords}

Insulin resistance $\cdot$ Beta-cell function · Graves' disease .

Carbimazole

\begin{abstract}
Background and Objective: Thyroid hormones play an important role in intermediate metabolism, and abnormal glucose tolerance is often observed in patients with hyperthyroidism. Several pathogenic mechanisms have been proposed as contributors. However, there is no conclusive evidence in the existing literature regarding the predominant underlying pathophysiology. Our objective was to determine the changes in insulin resistance parameters and beta-cell function in patients with Graves' disease following restoration of a euthyroid state. Methodology: This was an observational study with a before-after study design. Fortyfive treatment-naïve adults with Graves' diseases were included and 36 completed the study. An oral glucose tolerance test was performed at baseline and after 3 months of achieving a stable euthyroid state to assess glucose tolerance, insulin sensitivity, and beta-cell function. All patients were treated with antithyroid medication. The outcome measures studied were the Homeostasis Model Assess-
\end{abstract}

ment-2 Insulin Resistance (HOMA2-IR), Matsuda index, and Insulin Secretion-Sensitivity Index (ISSI)-2. Results: Twothirds of the patients had abnormal glucose tolerance at baseline. Among those with abnormal glucose tolerance at baseline, $34.7 \%$ had persistent abnormality during followup. During follow-up, no significant change was noted in the indices of insulin resistance. Patients with abnormal glucose tolerance had a significantly lower ISSI-2 index at baseline and it improved after achieving a euthyroid state. Conclusions: Abnormal glucose tolerance is a significant metabolic consequence in patients with Graves' disease. Decreased beta-cell function was observed among those with abnormal glucose tolerance and it improved during follow-up. In a proportion of patients, abnormal glucose tolerance persisted after 3 months, emphasizing the need for continued follow-up.

(C) 2019 European Thyroid Association Published by S. Karger AG, Basel

\section{Introduction}

Graves' disease (GD) is one of the most commonly encountered disorders in clinical practice with an annual incidence of $20-50$ cases per 100,000 persons [1]. Thyroid

\section{KARGER}

(C) 2019 European Thyroid Association

Published by S. Karger AG, Basel

E-Mail karger@karger.com

www.karger.com/etj
Sadishkumar Kamalanathan, Additional Professor and Head

Department of Endocrinology and Metabolism

4th Floor, Super Specialty Block

Puducherry 605006 (India)

E-Mailsadishkk@gmail.com 
hormones (THs) play a key role in intermediate metabolism, and abnormal glucose tolerance and diabetes mellitus have been reported to occur in a significant proportion of patients with hyperthyroidism [2]. Several factors like changes in insulin resistance, beta-cell function, abnormal gastric emptying, and intestinal absorption of glucose have been proposed as potential mechanisms that result in abnormal glucose homeostasis.

THs contribute to a state of insulin resistance in the liver and peripheral tissues through several mechanisms. They induce the expression of enzymes such as phosphoenolpyruvate carboxykinase (PCK1) and glucose-6-phosphatase catalytic subunit (G6PC), the key mediators of gluconeogenesis and glycogenolysis, and glucose transporter 2 (GLUT2) in the liver, which contributes to increased hepatic glucose output [3-6]. In adipose tissue, $\mathrm{TH}$ increases lipid oxidation [7] and augments the sensitivity of adipocyte lipolysis to catecholamines and indirectly increases fatty acid efflux [8]. In skeletal muscles, the rate of glycogen synthesis is reduced, and glucose, through conversion into lactate, is shunted to the Cori cycle in the liver. This results in increased glucose output. The rate of glycogenolysis has shown to be increased in animal models $[9,10]$. However, changes in insulin sensitivity/resistance in patients with GD have not been consistently demonstrated in the literature [11-13].

The other potential mechanism that can contribute to abnormal glucose tolerance is suboptimal beta-cell function. Studies measuring insulin secretion rates in response to stimulation by secretagogues like L-arginine and $\beta_{2}$-receptor agonists and oral glucose load have also yielded conflicting results [14-18].

The prime abnormality that contributes to abnormal glucose homeostasis in patients with GD and its changes with therapy remains unresolved. This study has thus been designed with the objective of determining the changes in insulin resistance and beta-cell function in patients with GD and the changes in these parameters after achieving a stable euthyroid state with medical therapy.

\section{Research Design and Methods}

Forty-five treatment-naïve patients diagnosed as having GD and presenting to the endocrinology and metabolism outpatient department at the Jawaharlal Institute of Postgraduate Medical Education and Research (JIPMER), Puducherry, India, were recruited for this study. Patients who were known to have prediabetes or diabetes mellitus, polycystic ovary syndrome, chronic kidney disease, hepatitis or cirrhosis, malignancy, or active infection, as well as those on long-term steroid therapy, were excluded. History-taking and a detailed clinical examination were performed on all participants. The participants were subjected to an oral glucose tolerance test (OGTT) as described below. They were then started on carbimazole tablets at the recommended dosage and were monitored with serial thyroid function tests at regular intervals. The OGTT was repeated after the patients had achieved a stable state of euthyroidism. A stable euthyroid state was defined, for the purpose of this study, as documentation of normal TH levels (free [f] $\mathrm{T}_{3}$ and $\mathrm{fT}_{4}$ ) twice, at least 3 months apart.

\section{Oral Glucose Tolerance Test}

All participants were subjected to a standard $75 \mathrm{~g}$ OGTT after an overnight fast for a minimum of $8 \mathrm{~h}$. Venous blood was collected in plain and fluoride tubes 0, 30, 60, and $120 \mathrm{~min}$ after glucose load for estimation of serum insulin and glucose, respectively. The blood samples were allowed to clot for 15-30 min and were centrifuged at 2,500 rpm for $10 \mathrm{~min}$. The serum samples were stored at a temperature of $-80^{\circ} \mathrm{C}$ until analysis.

\section{Biochemical and Hormonal Analysis}

Serum TSH, $\mathrm{fT}_{3}$ and $\mathrm{fT}_{4}$, and insulin were measured by competitive immunoassay using direct chemiluminescence technology (ADVIA Centaur XP). This assay measures TSH concentrations up to $150 \mathrm{mIU} / \mathrm{L}$ with a minimum detectable concentration of $0.010 \mathrm{mIU} / \mathrm{L}$. The $\mathrm{fT}_{4}$ assay measures $\mathrm{fT}_{4}$ concentrations up to 12.0 $\mathrm{ng} / \mathrm{dL}$, with an analytical sensitivity of $0.1 \mathrm{ng} / \mathrm{dL}$. The $\mathrm{fT}_{3}$ assay can measure $\mathrm{fT}_{3}$ concentrations up to $20 \mathrm{pg} / \mathrm{mL}$, with a minimum detectable concentration of $0.2 \mathrm{pg} / \mathrm{mL}$. The insulin assay measures insulin concentrations up to $300 \mathrm{mU} / \mathrm{L}$, with a minimum detectable concentration of $0.5 \mathrm{mU} / \mathrm{L}$. Plasma glucose was estimated by the glucose oxidase method.

\section{Insulin Sensitivity/Resistance Indices}

Homeostasis Model Assessment-2 Insulin Resistance (HOMA2-IR) was calculated using the computer software (HOMA Calculator v2.2.2) [19]. The Matsuda index was calculated as described by Matsuda and DeFronzo [20]. The area under the curve (AUC) for insulin and glucose was calculated by the linear trapezoidal method. The Insulin Secretion-Sensitivity Index -2 (ISSI-2) was calculated as a product of (AUC insulin/AUC glucose) and the Matsuda index. This index has been shown to have a modestly strong correlation with the disposition index determined by the frequently sampled intravenous glucose tolerance test [21].

\section{Statistical Analysis}

All statistical analysis was performed using the Statistical Package for the Social Sciences version 19.0 (SPSS). Continuous variables are presented as the mean \pm standard deviation or median with range, depending on the distribution of the variable. Normality of the data was assessed by the Shapiro-Wilk test. The paired $t$ test and Wilcoxon signed-rank test were used to compare parameters before and after achieving a stable euthyroid state. The independent Student $t$ test and Mann-Whitney $\mathrm{U}$ test were conducted to compare two independent groups. Appropriate tests were chosen based on the normality of the data. A $p$ value $<0.05$ was considered significant.
Lakshmana Perumal/Selvi/Sridharan/ Sahoo/Kamalanathan 


\section{Results}

Clinical, Anthropometric, and Hormonal Parameters

Forty-five patients who were diagnosed as having GD during the study period were included in the study. One patient developed acute viral hepatitis and another patient opted for radioiodine ablation after inclusion in the study; both patients were hence excluded. Seven participants were lost to follow-up. Thirty-six of the participants finally completed the study. Three-fourths of the participants were women and the average age of the patients was $36.3 \pm 9.1$ years. The mean duration of symptoms of hyperthyroidism prior to diagnosis was 6.8 months. The mean body mass index (BMI) at baseline was $18.54 \pm 2.39$, which rose to $21.1 \pm 2.87(p<0.001)$ following the establishment of a euthyroid state. The average $\mathrm{fT}_{3}$ value at baseline was $18.83 \pm 7.75 \mathrm{pg} / \mathrm{mL}$ and that of $\mathrm{fT}_{4}$ was $5.18 \pm 3.25 \mathrm{ng} / \mathrm{dL}$.

\section{Plasma Glucose Abnormalities Detected during the OGTT}

At baseline, $13.8 \%$ of the patients had impaired fasting glucose (IFG), 33\% had impaired glucose tolerance (IGT), and $11.1 \%$ had both IFG and IGT. 5.5\% of the patients had blood glucose levels in the range of diabetes mellitus. During follow-up, $11.1 \%$ had IFG, IGT was detected in $11.4 \%$ of the patients, and 5.5\% had both IFG and IGT. $2.7 \%$ had diabetes mellitus. Of those with normal glucose tolerance at baseline, 3 had abnormal glucose tolerance at follow-up; 1 patient had IFG and 2 patients had IGT. The patients with normal glucose tolerance had higher $\mathrm{fT}_{4}$ levels at baseline when compared to those with abnormal glucose tolerance $(6.19 \pm 3.19$ vs. $4.70 \pm 4.92 \mathrm{ng} / \mathrm{dL} ; p=0.04)$. There was no other significant difference in family history of type 2 diabetes mellitus and anthropometric and thyroid function test parameters between the two groups.

\section{Changes in Insulin Resistance/Sensitivity Indices}

There was no significant change in HOMA2-IR and Matsuda index during follow-up in the whole study cohort (Table 1). There was also no significant difference noted during follow-up in these parameters in subgroup analyses of those with normal and those with abnormal glucose tolerance (Table 1). Also, there was no difference in either parameter between those with abnormal and those with normal OGTT results at baseline.

\section{Changes in ISSI-2}

There was no significant change noted in ISSI-2 after restoration of a euthyroid state in the whole study cohort (Table 1). However, the ISSI-2 was significantly lower
Table 1. Changes in insulin sensitivity/resistance and beta-cell function at baseline and follow-up

\begin{tabular}{lccc}
\hline Parameter & Baseline & Follow-up & $p$ value \\
\hline Whole study cohort $(n=36)$ & & \\
HOMA2-IR & $1.19 \pm 0.78$ & $1.33 \pm 1.02$ & 0.86 \\
Matsuda index & $3.18 \pm 2.53$ & $3.52 \pm 2.43$ & 0.64 \\
ISSI-2 & $1.64 \pm 0.81$ & $1.81 \pm 0.92$ & 0.09 \\
\hline Patients with normal OGTT results at baseline $(n=13)$ & \\
HOMA2-IR & $1.22 \pm 0.50$ & $1.21 \pm 0.59$ & 0.91 \\
Matsuda index & $3.97 \pm 3.03$ & $3.83 \pm 2.57$ & 0.46 \\
ISSI-2 & $1.97 \pm 0.62$ & $2.02 \pm 0.56$ & 0.82 \\
\hline Patients with abnormal OGTT results at baseline $(n=23)$ & \\
HOMA2-IR & $1.27 \pm 0.71$ & $1.35 \pm 1.05$ & 0.53 \\
Matsuda index & $2.79 \pm 1.83$ & $3.01 \pm 1.57$ & 0.95 \\
ISSI-2 & $1.39 \pm 0.58$ & $1.79 \pm 1.15$ & $0.02 *$ \\
\hline
\end{tabular}

Values are presented as the mean \pm standard deviation. HOMA2-IR, Homeostasis Model Assessment-2 Insulin Resistance; ISSI-2, Insulin Secretion-Sensitivity Index-2; OGTT, oral glucose tolerance test. ${ }^{*}$ Significant.

among those with abnormal glucose tolerance at baseline than among those with normal glucose tolerance (1.39 \pm 0.58 vs. $1.97 \pm 0.62 ; p=0.02$ ). There was also a significant improvement in this parameter during follow-up among those with abnormal glucose tolerance (Table 1).

\section{Discussion}

Disturbance of glucose tolerance and worsening of diabetes occur in a significant number of patients with hyperthyroidism. Two-thirds of our patients had abnormal glucose tolerance at baseline, which included 2 patients with plasma glucose abnormalities in the range of diabetes mellitus. The reported incidence of abnormal glucose tolerance in the literature varies from 39.4 to $57 \%$. In the study by Roubsanthisuk et al. [18], those with abnormal glucose tolerance had higher $\mathrm{fT}_{4}$ levels at baseline, in contrast to what was observed in our study. Apart from this observation, there were no other significant differences in any of the baseline parameters studied.

Even after 3 months of maintaining a stable euthyroid state, one-third of those with abnormal glucose tolerance at baseline continued to demonstrate abnormalities during the follow-up OGTT. In the study by Maxon et al. [22], abnormal glucose tolerance persisted in around $40 \%$ 
of their patients 9 months after achieving a euthyroid state. This indicates that disturbances in glucose homeostasis may take much longer to return to baseline, and hence patients with hyperthyroidism and abnormal glucose tolerance may need to be closely monitored for a longer duration.

Insulin resistance at the level of the liver and peripheral tissues has been documented in many studies. A significant improvement in insulin resistance has been documented with resolution of the hyperthyroid state. Maratou et al. [12] demonstrated increased insulin resistance in patients with clinical and subclinical hyperthyroidism when compared to euthyroid controls. The average BMI of the patients with hyperthyroidism in this study was $24.85 \pm 0.8$, compared to $18.54 \pm 2.39$ in our study.

Chu et al. [23] reported an elevated HOMA-IR in 19 patients with hyperthyroidism compared to healthy controls, which decreased after normalization of the thyroid status. However, in our study, no difference in hepatic (assessed by HOMA2-IR) or whole-body insulin sensitivity (assessed by Matsuda index) was noted during followup. Recently, in the study by Chng et al. [13], no significant change in HOMA-IR was noted within 4 weeks of achieving a euthyroid state in patients with GD.

Differences in the duration and severity of hyperthyroidism, in addition to other traditional risk factors like age, BMI, ethnicity, and a family history of diabetes mellitus could contribute to the variations in incidence of abnormal glucose tolerance and insulin resistance noted across these studies. Also, there is a significant increase in body weight following restoration of the euthyroid state, which could explain the absence of improvement in insulin resistance/sensitivity parameters.

In the current study, beta-cell function (assessed by ISSI-2) was found to be impaired among those with abnormal glucose tolerance when compared to those with normal glucose tolerance. There was also a significant improvement at follow-up, which could explain the improvement in glucose homeostasis noted in these patients.

Ikeda et al. [24] demonstrated that insulin secretion in proportion to a blood sugar stimulus was significantly reduced in hyperthyroid patients, and that this abnormality normalized with the achievement of a euthyroid state. However, insulin secretion during intravenous glucose tolerance testing was not impaired, suggesting a possible role of incretins in the impaired insulin secretion during the OGTT. Bech et al. [7] studied beta-cell function in 9 untreated patients using a mixed-meal test. The mean insulin secretory capacity was reduced by $50 \%$ in their patients with hyperthyroidism when compared to controls.
A few animal studies have offered insights into how hyperthyroidism could influence beta-cell function. A reduced volume of pancreatic islets and a lower number of insulin-positive cells have been demonstrated in rats with experimentally induced hyperthyroidism. A defective insulin secretory response due to a possible defect in $\mathrm{K}_{\mathrm{ATP}}$ and L-type calcium channels was also noted [25]. Similarly, severe hyperthyroidism has also been shown to induce apoptosis of beta cells and to accelerate the rate of apoptosis of pancreatic ductal cells, which serve as precursors of beta cells. This effect was largely reversible with time, and a normalization of the beta-cell volume was noted due to an increased cell replication rate when the effect of thyroxine was waning $[26,27]$.

The strengths of the present study are as follows: (1) changes in indices of hepatic insulin resistance and wholebody insulin sensitivity have been studied to assess different aspects of the effects of TH; (2) the ISSI-2 used to assess beta-cell function is a composite measure that accounts for insulin resistance and blood glucose values while assessing beta-cell function, thus making it a better marker than indices that utilize only insulin or C-peptide and glucose values; and (3) follow-up measurement of outcome variables was done after 3 months of maintaining euthyroidism, to allow for the stabilization of disturbed pathophysiological factors.

The limitations of the current study are as follows: (1) the OGTT is not the gold standard for assessing insulin sensitivity/resistance; however, it is considered an acceptable alternative as it is not feasible to perform clamp studies outside the premises of certain research facilities; (2) healthy matched controls and patients with non-autoimmune-induced thyroid dysfunction (toxic nodular goiter) were not included in the study, making it difficult to draw conclusions about baseline parameters; (3) although a period of 3 months was allowed to elapse after achieving a euthyroid state, one-third of the study population still had some abnormality of glucose tolerance; hence, reassessment of patients after a longer follow-up period might be necessary; and (4) the findings of the study may not be applicable to those being treated with other modalities like radioiodine ablation or surgery.

To conclude, abnormal glucose tolerance is a metabolic consequence of hyperthyroidism observed in a majority of patients. The current study found no significant change in insulin sensitivity/resistance indices after restoration of a euthyroid state in patients with GD. However, patients with abnormal glucose tolerance had a significantly lower beta-cell function than those with normal glucose tolerance, and it improved significantly after
Lakshmana Perumal/Selvi/Sridharan/ Sahoo/Kamalanathan 
achieving a stable euthyroid state. In a subset of patients with abnormal glucose tolerance, the metabolic abnormality persisted 3 months after restoration of a euthyroid state. This indicates that those with abnormal glucose tolerance need longer follow-up to better understand the clinical course of the metabolic derangements induced by hyperthyroidism.

\section{Statement of Ethics}

All procedures performed in this study were in accordance with the ethical standards of the institutional and national research committee and with the 1964 Helsinki Declaration and its later amendments or comparable ethical standards. Informed consent was obtained from all individual participants included in the study.

\section{Disclosure Statement}

The authors have no conflicts of interest to declare.

\section{Funding Sources}

This study was entirely supported by intramural funding from JIPMER (project No. JIP/Res/Intra-DM-M.Ch/01/2015-2016).

\section{References}

1 Smith TJ, Hegedüs L. Graves' disease. N Engl J Med. 2016 Oct;375(16):1552-65.

2 Paul DT, Mollah FH, Alam MK, Fariduddin M, Azad K, Arslan MI. Glycemic status in hyperthyroid subjects. Mymensingh Med J. 2004 Jan;13(1):71-5.

3 Suh JH, Sieglaff DH, Zhang A, Xia X, Cvoro A, Winnier GE, et al. SIRT1 is a direct coactivator of thyroid hormone receptor $\beta 1$ with gene-specific actions. PLoS One. 2013 Jul; 8(7):e70097.

4 Park EA, Song S, Vinson C, Roesler WJ. Role of CCAAT enhancer-binding protein beta in the thyroid hormone and cAMP induction of phosphoenolpyruvate carboxykinase gene transcription. J Biol Chem. 1999 Jan;274(1): 211-7.

5 Weinstein SP, O'Boyle E, Fisher M, Haber RS. Regulation of GLUT2 glucose transporter expression in liver by thyroid hormone: evidence for hormonal regulation of the hepatic glucose transport system. Endocrinology. 1994 Aug;135(2):649-54.

6 Mokuno T, Uchimura K, Hayashi R, Hayakawa N, Makino M, Nagata M, et al. Glucose transporter 2 concentrations in hyper- and hypothyroid rat livers. J Endocrinol. 1999 Feb;160(2):285-9.

7 Bech K, Damsbo P, Eldrup E, Beck-Nielsen H, Røder ME, Hartling SG, et al. Beta-cell function and glucose and lipid oxidation in Graves' disease. Clin Endocrinol (Oxf). 1996 Jan;44(1):59-66.

8 Goodman HM, Knobil E. Mobilization of fatty acids by epinephrine in normal and hypophysectomized rhesus monkeys. Proc Soc Exp Biol Med. 1959 Jan;100(1):195-7.

9 Dimitriadis G, Parry-Billings M, Bevan S, Leighton B, Krause U, Piva T, et al. The effects of insulin on transport and metabolism of glucose in skeletal muscle from hyperthyroid and hypothyroid rats. Eur J Clin Invest. 1997 Jun;27(6):475-83.
10 Dimitriadis GD, Leighton B, Vlachonikolis IG, Parry-Billings M, Challiss RA, West D, et al. Effects of hyperthyroidism on the sensitivity of glycolysis and glycogen synthesis to insulin in the soleus muscle of the rat. Biochem J. 1988 Jul;253(1):87-92.

11 Kapadia KB, Bhatt PA, Shah JS. Association between altered thyroid state and insulin resistance. J Pharmacol Pharmacother. 2012 Apr;3(2):156-60.

12 Maratou E, Hadjidakis DJ, Peppa M, Alevizaki M, Tsegka K, Lambadiari V, et al. Studies of insulin resistance in patients with clinical and subclinical hyperthyroidism. Eur J Endocrinol. 2010 Oct;163(4):625-30.

13 Chng CL, Lim AY, Tan HC, Kovalik JP, Tham KW, Bee YM, et al. Physiological and metabolic changes during the transition from hyperthyroidism to euthyroidism in Graves' disease. Thyroid. 2016 Oct;26(10): 1422-30.

14 Seino Y, Goto Y, Taminato T, Ikeda M, Imura $\mathrm{H}$. Plasma insulin and glucagon responses to arginine in patients with thyroid dysfunction. J Clin Endocrinol Metab. 1974 Jun;38(6): $1136-40$.

15 Cavagnini F, Peracchi M, Raggi U, Bana R, Pontiroli AE, Malinverni A, et al. Impairment of growth hormone and insulin secretion in hyperthyroidism. Eur J Clin Invest. 1974 Feb; 4(1):71-7.

16 Ahrén B, Lundquist I, Hedner P, Valdemarsson S, Scherstén B. Glucose tolerance and insulin and C-peptide responses after various insulin secretory stimuli in hyper- and hypothyroid subjects before and after treatment. Diabetes Res. 1985 Mar;2(2):95-103.

17 Bonora E, Manicardi V, Zenere M, Moghetti P, Coscelli C, Muggeo M. Plasma C-peptide response to oral glucose load in hyperthyroidism. J Endocrinol Invest. 1990 Jul-Aug;13(7):555-8.
18 Roubsanthisuk W, Watanakejorn P, Tunlakit M, Sriussadaporn S. Hyperthyroidism induces glucose intolerance by lowering both insulin secretion and peripheral insulin sensitivity. J Med Assoc Thai. 2006 Nov;89 Suppl 5:S133-40.

19 Levy JC, Matthews DR, Hermans MP. Correct Homeostasis Model Assessment (HOMA) evaluation uses the computer program. Diabetes Care. 1998 Dec;21(12):2191-2.

20 Matsuda M, DeFronzo RA. Insulin sensitivity indices obtained from oral glucose tolerance testing: comparison with the euglycemic insulin clamp. Diabetes Care. 1999 Sep;22(9):1462-70.

21 Retnakaran R, Qi Y, Goran MI, Hamilton JK. Evaluation of proposed oral disposition index measures in relation to the actual disposition index. Diabet Med. 2009 Dec;26(12):1198-203.

22 Maxon HR, Kreines KW, Goldsmith RE, Knowles HC Jr. Long-term observations of glucose tolerance in thyrotoxic patients. Arch Intern Med. 1975 Nov;135(11):1477-80.

23 Chu CH, Lam HC, Lee JK, Lu CC, Sun CC, Wang MC, et al. Hyperthyroidism-associated insulin resistance is not mediated by adiponectin levels. J Thyroid Res. 2011 Jan;2011:194721.

24 Ikeda T, Fujiyama K, Hoshino T, Takeuchi T, Mashiba H, Tominaga M. Oral and intravenous glucose-induced insulin secretion in hyperthyroid patients. Metabolism. 1990 Jun; 39(6):633-7.

25 Karbalaei N, Noorafshan A, Hoshmandi E. Impaired glucose-stimulated insulin secretion and reduced $\beta$-cell mass in pancreatic islets of hyperthyroid rats. Exp Physiol. 2016 Aug;101(8):1114-27.

26 Ximenes HM, Lortz S, Jörns A, Lenzen S. Triiodothyronine (T3)-mediated toxicity and induction of apoptosis in insulin-producing INS-1 cells. Life Sci. 2007 May;80(22):2045-50.

27 Jörns A, Tiedge M, Lenzen S. Thyroxine induces pancreatic beta cell apoptosis in rats. Diabetologia. 2002 Jun;45(6):851-5. 